\title{
Correlated changes in body shape after five generations of selection to improve growth rate in a breeding program for Nile tilapia Oreochromis niloticus in Brazil
}

\author{
Carlos Antonio Lopes de Oliveira $^{1} \cdot$ Ricardo Pereira Ribeiro $^{1}$. \\ Grazyella Massako Yoshida ${ }^{2}$. Natali Miwa Kunita ${ }^{3}$ - Gabriel Soriani Rizzato ${ }^{3}$. \\ Sheila Nogueira de Oliveira ${ }^{4}$. Alexandra Inês dos Santos ${ }^{5}$. Nguyen Hong Nguyen ${ }^{6}$
}

Received: 4 June 2015 /Revised: 12 October 2015 / Accepted: 8 January 2016 / Published online: 26 January 2016

(C) Institute of Plant Genetics, Polish Academy of Sciences, Poznan 2016

\begin{abstract}
Body shape is a commercial trait of great interest as it impacts profit and productivity of aquaculture enterprises. In the present study, we examined correlated changes in two measures of body shape (depth to length ratio, DL-R and ellipticity of mid sagittal plane, EL-H) from a selection program for high daily weight gain in a Nile tilapia population reared in freshwater cages in Brazil. Genetic parameters for body shape and its genetic association with growth traits (body weight and daily gain) were also estimated from 8 , 725 individuals with growth performance recorded over five generations from 2008 to 2013. Mixed model analysis showed that the selection program resulted in substantial improvement in growth performance (about $4 \%$ genetic gain per generation
\end{abstract}

Communicated by: Maciej Szydlowski

Carlos Antonio Lopes de Oliveira

caloliveira@uem.br

1 Universidade Estadual de Maringá, Avenida Colombo, 5790, Jardim Universitário, 87020-900 Maringá, Paraná, Brazil

2 Universidade Estadual Júlio Mesquita Filho - FCAV/Jaboticabal, Via de Acesso Prof. Paulo Donato Castellane s/n, 14884-900 Jaboticabal, SP, Brazil

3 Aquaamerica Company, Rodovia BR 491 — km 158 Fazenda Porto, 37130-000 Alfenas, Minas Gerais, Brazil

4 Faculdade de Ciências Agrárias Universidade Federal da Grande Dourados, Caixa Postal - 533, 79.804-970 Dourados, Mato Grosso do Sul, Brazil

5 Universidade Federal da Bahia, Escola de Medicina Veterinária e Zootecnia, Avenida Adhemar de Barros, Ondina, 40170-110 Salvador, Bahia, Brazil

6 The University of the Sunshine Coast, Locked Bag 4, Maroochydore DC, QLD 4558, Australia or per year) and also brought about trivial changes in body shape. The heritabilities ranged from 0.470 to 0.564 for growth traits and 0.180 to 0.289 for body shape. The common family effects were low for all traits studied, accounting for only $3-11 \%$ of total phenotypic variance. The genetic correlations between body shape and growth traits were weak, i.e., -0.385 between EL-H and growth traits and 0.28 between DL-R and body weight or daily gain. Strong and negative genetic association was found between the two body shape traits $(\mathrm{rg}=--0.955)$. Harvest body weight and daily gain are essentially the same traits, as indicated by the close to one genetic correlations between the two characters. Our results demonstrated that the selection process to increase growth rate had small, but slowly constant effect in body shape traits; and in the long term, the fish would have become rotund.

Keywords Heritability · Genetic correlation · Daily gain · Rotund fish $\cdot$ Body depth

\section{Introduction}

Fish body shape is one of the commercially important traits that determines market values of an animal and thus economic return for aquaculture enterprises. This is because body shape traits are related to animal appearance (Colihueque and Araneda 2014) and consumer preference (Kause et al. 2003; Nguyen et al. 2007). The shape of the fish are also correlated with fillet weight and fillet quality; the round fish having greater yield than their skinny and long body counterparts (Haffray et al. 2013; Whatmore et al. 2013).

Across the aquaculture sector, there has been a growing interest in understanding quantitative genetic basis of body shape. This trait is, however, not directly and easily measured, 
and it is often calculated using measurements of body dimensions as a ratio of weight over length or depth over length (Weatherley et al. 1987). Some other studies also examined body shape in fish by using geometric morphometric image analysis such as in common sole (Blonk et al. 2010), European sea bass (Costa et al. 2010), or rainbow trout (Komen et al. 2014). Regardless of trait definitions and measurement methods, several reports showed that there is an additive genetic component for this trait, with the heritability estimate ranging from 0.08 to 0.25 across farmed aquaculture species (Gjerde and Schaeffer 1989; Trọng et al. 2013; Komen et al. 2014). The low heritability for body shape, however, suggests that selection to change the fish body shape may be slow. Theoretical predictions using selection index approach pointed out that selection for high growth or increased body weight, albeit at a very slow rate, can cause correlated changes in tilapia body shape in the long term (Nguyen et al. 2007).

To date, there are, however, no reports on realized correlated changes in body shape from selection programs for high growth in fish or other aquatic animal species. Based on genetic correlation estimates, a few studies (Trọng et al. 2013) have predicted that selection for high growth can produce round body shape fish.

In the present study, we attempted to address three main questions: i) the effect of selection for high growth rate on tilapia body shape, ii) the genetic association between growth rate and body shape traits, and iii) is there sufficient genetic variation in body shape traits to justify a breeding program? Specific objectives of our study were to estimate the variance components and heritabilities, genetic and phenotypic correlations between growth rate and body shape traits, and to evaluate the correlated genetic response in body shape from the selection program for high daily weight gain over five generations (2008-2013) in a Nile tilapia population cultured in a cage environment in Brazil.

\section{Material and methods}

\section{The population}

The population established in Brazil originated from the genetically improved farmed tilapia (GIFT) strain. In 2006, a total of 600 fish (20 individuals $\times 30$ families) were imported to State University of Maringá, Brazil. These animals have been used to form a base population for continuing selection since 2007.

In each generation, between 37 and 74 full- and half-sib families were produced and performance tested in freshwater cages at the Corvo River, Diamante do Norte, Paraná State, Brazil. The mating system was hierarchical, one male and two females, as described by Santos et al. (2011).The reproduction season occurred from November to March annually.
The performance test was conducted from the autumn to spring (April to October) in all spawning years except that the last generation (2013) was evaluated from summer to winter (February-August). During the grow-out testing period, the water temperature and dissolved oxygen were in the range $19.0-26.9^{\circ} \mathrm{C}$ and $3.8-6.8 \mathrm{mg} \mathrm{l}^{-1}$ respectively. The stocking density in grow-out cages was $100 \mathrm{~kg} \mathrm{~m}^{-3}$.In the first 30 days of culture, the diet was dry pellet feed with $45 \%$ crude protein provided to the fish three times a day; thereafter, the dry pellets with $32 \%$ crude protein were provided twice a day until harvest.

After harvest and measurements of body dimensions, the growth data were processed to estimate breeding values (EBV) for all individuals in the pedigree. The highest EBV animals within families were selected to become parents to produce next generations; all the families were represented in mating groups. On average, $15.8 \%$ of females and $7 \%$ of males were selected in each generation, corresponding to a selection intensity of 1.51 and 1.93 respectively. The selection criteria were body weight at harvest in the first generation (year) and daily gain in subsequent years. Approximately 120 females and 60 males were used to produce the next generation. The same selection and breeding procedures were repeated for all generations from 2008 to 2013.

\section{Traits}

After the grow-out period of about 6 months, measurements were taken of live weight (W), standard (SL) and total body length (TL), head length (HL), and body depth (BD) (Kunita et al. 2013).

For this study, we examined two groups of traits:1) growth performance and 2) body shape. The performance traits were weight (W) and daily gain (DG). Daily gain was calculated using the following formula, $D G=\frac{w}{a g e}$, where $\mathrm{w}=$ harvest weight and age from stocking to harvest.

The body shape traits were depth to length ratio (DL-R) and ellipticity of the mid sagittal plane (EL-H). The depth to length ratio values were calculated using $D L-R=\left(\frac{B D}{B L}\right)-0.5$, where $\mathrm{BL}$ is the body length, $B L=S L-H L, 0.5$ is arbitrary value considering $B L=2(B D)$. A positive deviation from 0.5 indicate a round-shaped fish.

The ellipticity of the mid-sagittal plane (EL-H) was calculated following Trọng et al. (2013) as $E L-H=\frac{S L-B D}{S L+B D}$. A small value for EL-H represents a circular (round) shape, whereas a large value towards one indicates an elongated shape. A perfect circle has an ellipticity value of zero.

\section{Data set}

The growth and body shape data were collected over five generations (2008 - 2013) from the selection program for high 
growth in Nile tilapia (GIFT strain origin) under freshwater cages. The data set contained 8,725 data records collected from 8,906 animals. The pedigree information, data structure, and basic descriptive statistics are described in Table 1.

\section{Statistical models}

Preliminary analyses using general linear model, and likelihood ratio test were performed to determine the presence of systematic effects and random terms in the model. The final statistical model used to analyse body shape and growth traits was written in mathematic notations as follows:

$y_{i j k l m}=\mu+c_{i}+s_{j}+b_{1} a_{k}+b_{2} a_{k}^{2}+f_{l}+a_{i j k l m}$

where $y_{i j k l m}$ is the animal observation, $\mu$ is general constant, $c_{i}$ is the cage within generation effect, $s_{j}$ is sex effect, $a_{k}$ is age from birth to harvest, $b_{1}$ and $b_{2}$ linear and quadratic coefficients, $f_{l}$ is a family common effect, $a_{i j k l m}$ is the additive genetic effect of individual fish in the pedigree, and $e_{i j k l m}$ is the error term.

\section{Genetic parameters}

Bayesian inference (Sorensen and Gianola 2002) applied to a single trait mixed model analysis was performed to estimate the variance components and heritability and family common effects for traits studied, including body weight (W), daily gain (DG), ellipticity of mid sagittal plane (EL-H), and depth to length ratio (DL-R).

The phenotypic and genetic correlations between traits were estimated using a two-trait model with the same fixed and random effects as described in Eq. 1. The two-trait analyses involved a combination of all pair-wise traits: $\mathrm{W}$ and $\mathrm{DG}$, DL-R and EL-H.

The (co)-variance components and genetic parameters were estimated using a computational system MTGSAM (multiple trait Gibbs sampler for animal models) (Van Tassell and Van Vleck 1995).The strategy to conduct single trait analysis was 250,000,50,000, and 50, by total, burn-in, and thinning interval cycles, resulting in chains with 4,000 samples. For two-trait analysis the chains had 20,000 samples, from 550,000, 50,000 and 25, by total, burn-in, and thinning interval cycles.

The chains convergence was evaluated by the Heidelberger and Welch method (Cowles and Carlin 1996) using the

Table 1 General information about the data set, means, standard deviation (parenthesis), and range (second line)

\begin{tabular}{|c|c|c|c|c|c|}
\hline & \multicolumn{5}{|l|}{ Generations } \\
\hline & 1 & 2 & 3 & 4 & 5 \\
\hline$N$ sires & 24 & 40 & 52 & 39 & 29 \\
\hline$N$ dams & 33 & 57 & 79 & 44 & 42 \\
\hline$N$ total & 1731 & 1717 & 2695 & 1127 & 1455 \\
\hline$N$ males $/ N$ females & 0.932 & 1.302 & 0.919 & 0.971 & 1.425 \\
\hline Days in test & 251 & 168 & 170 & 218 & 232 \\
\hline \multirow[t]{2}{*}{ Progeny by sire $^{1}$} & $72.2(32.1)$ & $42.9(18.9)$ & $51.8(17.6)$ & $28.9(19.0)$ & $50.2(26.5)$ \\
\hline & $28-30$ & $12-92$ & $17-79$ & $3-74$ & $15-19$ \\
\hline \multirow[t]{2}{*}{ Progeny by dam ${ }^{1}$} & $52.5(12.9)$ & $30.1(10.8)$ & $34.1(6.3)$ & $25.6(13.0)$ & $34.6(13.7)$ \\
\hline & $28-70$ & $8-70$ & $15-53$ & $3-61$ & $12-69$ \\
\hline \multirow[t]{2}{*}{ Weight (g) } & $792.3(278.3)$ & $262.4(83.1)$ & $402.8(140.8)$ & $552.9(257.5)$ & $780.3(328.7)$ \\
\hline & $182-1,518$ & $51-653$ & $54-906$ & $56-1,682$ & $81-1,755$ \\
\hline \multirow[t]{2}{*}{ Daily gain (g/day) } & $1.99(0.71)$ & $0.92(0.28)$ & $1.37(0.47)$ & $1.80(0.81)$ & $2.66(1.03)$ \\
\hline & $0.49-3.76$ & $0.18-2.70$ & $0.18-3.09$ & $0.17-4.90$ & $0.31-5.70$ \\
\hline \multirow[t]{2}{*}{ DL-R } & $0.051(0.033)$ & $0.052(0.037)$ & $0.059(0.045)$ & $0.062(0.045)$ & $0.091(0.045)$ \\
\hline & -0.161 to 0.431 & -0.228 to 0.349 & -0.224 to 0.629 & -0.313 to 0.478 & -0.387 to 0.442 \\
\hline \multirow[t]{2}{*}{ EL-H } & $0.449(0.021)$ & $0.448(0.022)$ & $0.446(0.026)$ & $0.443(0.027)$ & $0.420(0.028)$ \\
\hline & $0.242-0.626$ & $0.325-0.684$ & $0.255-0.672$ & $0.298-0.722$ & $0.276-0.853$ \\
\hline \multirow[t]{2}{*}{ Age (days) } & 396.7 (11.4) & $284.7(21.4)$ & $294.8(12.2)$ & $308.7(33.2)$ & $289.3(18.32)$ \\
\hline & $373-412$ & 231-309 & $234-314$ & $236-367$ & $259-316$ \\
\hline
\end{tabular}

$N=$ number; $\bar{X}=$ average, DL-R = depth to length ratio and EL-H = ellipticity of the mid sagittal plan

1. mean of evaluated individuals by sire or dam 
CODA v. 0.40 (convergence diagnosis and output analysis).The posterior means and the $95 \%$ credibility interval were calculated using $\mathrm{R}$ system v. 3.0.1.

\section{Genetic trends and selection response}

Estimated breeding values (EBV) were calculated using single-trait animal and common full-sib model (Eq. 1). Genetic trends were estimated by linear regression analyses of individual EBV on year of birth or generation. All the analyses were performed using the PROC Genmod in the SAS system version 9.0 (SAS Institute Inc 2007).

The selection response was also expressed as a percentage, and was calculated as the ratio of linear regression coefficient to least square means for the four traits studied.

\section{Results}

\section{Data structure and characteristics of the population}

Pedigree information (number of sires, dams, and offspring), data structure (number of females and males per family, and sex ratio), and the raw means for growth and body shape traits are given in Table 1. Over five generations of selection from 2008 to 2013, a total of 8,906 animals had growth and body shape recorded. The fish were harvested at an average body weight of about $560 \mathrm{~g}$ across generations. The average daily gain was $1.75 \mathrm{~g}$. The positive value for DL-R indicates that the fish show a rotund body shape. For ellipticity mid-sagittal plane $($ EL-H), the close to zero value is an indication of round-shaped fish.

\section{Heritability, family common effect, and phenotypic variance}

The heritability for the growth performance traits was high ( 0.469 and 0.489 for daily gain and weight), whereas those estimates were moderate for the body shape traits $(0.189$ to 0.198 for DL-R and EL-H respectively) (Table 2).

The family common effect $\left(\mathrm{c}^{2}\right)$ accounted for $3-11 \%$ of total variance (Table 2). The $\mathrm{c}^{2}$ estimates were greater for growth performance $(0.069$ and 0.112 for weight and daily gain) than for body-shape traits $\left(c^{2}=3-4 \%\right)$.

The small credibility interval and standard deviation (Table 2) indicated that all the estimates are statistically significant.

\section{Genetic and phenotypic correlations}

The genetic correlation between body weight and daily gain was high and positive $(>0.95)$. On the other hand, the estimate of genetic correlations between DL-R and ellipticity of the mid-sagittal plane (EL-H) was high and negative $(-0.95)$. The phenotypic associations between growth traits or between measures of body shape had similar sign and magnitude to the genetic correlations (Table 3).

The genetic and phenotypic associations between growth traits and measures of body shape were generally weak. The genetic correlation estimates of daily gain and weight with DL-R were positive but low (0.28), and negative ( -0.39$)$, with mid-sagittal ellipticity (Table 3 ).

\section{Genetic trends}

The genetic trend estimates were significant $(P<0.05)$ for all traits (Table 4). The genetic gain for performance traits (weight and daily gain) to selection was about $4 \%$ per generation (one year/generation). The regression analysis of genetic trends for body shape was significant, but the EBV gain/generation was small. A correlated increase in DL-R was only $0.31 \%$ per generation, and a correlated decrease in EL-H by $-0.09 \%$ per generation (Table 4).

\section{Discussion}

The foremost important aim of our study was to investigate if there are any correlated changes in body shape from the selection program for high growth in Nile tilapia. Genetic changes given in Table 4 showed that our selection program remarkadly improved body weight and daily gain by about $4 \%$ per generation, and also produced slight changes in body shape. The direct genetic response in daily gain falls within the range reported for other aquaculture species (Nguyen
Table 2 The posterior means, standard deviation (parenthesis) and credibility interval (second line) of heritability $\left(\mathrm{h}^{2}\right)$ and common full-sib effects $\left(c^{2}\right)$ for harvest weight, daily gain, DL-R and EL-H.

\begin{tabular}{lllll}
\hline & Weight & Dailygain & DL-R & EL-H \\
\hline $\mathrm{h}^{2}$ & $0.485(0.05)$ & $0.469(0.05)$ & $0.189(0.04)$ & $0.198(0.03)$ \\
& $0.384-0.589$ & $0.378-0.557$ & $0.126-0.265$ & $0.139-0.268$ \\
$\mathrm{c}^{2}$ & $0.069(0.02)$ & $0.112(0.02)$ & $0.035(0.01)$ & $0.029(0.01)$ \\
& $0.042-0.101$ & $0.079-0.149$ & $0.017-0.058$ & $0.014-0.049$ \\
$\sigma_{p}^{2}$ & $28,810(1051)$ & $0.276(0.0098)$ & $0.0017(0.00004)$ & $0.0006(0.00001)$ \\
& $26,888-30,990$ & $0.258-0.296$ & $0.0016-0.0018$ & $0.00057-0.00062$ \\
\hline
\end{tabular}


Table 3 The posterior means, standard deviation (parenthesis), and credibility interval of genetic (above) and phenotypic (below) correlations between performance and body-shape traits

\begin{tabular}{lllll}
\hline Traits & Weight & Daily gain & DL-R & EL-H \\
\hline Weight & & $0.983(0.003)$ & $0.281(0.112)$ & $-0.386(0.102)$ \\
& & $0.977-0.988$ & $0.059-0.488$ & -0.569 to 0.168 \\
Daily gain & $0.981(0.0009)$ & & $0.281(0.108)$ & $-0.385(0.096)$ \\
& $0.979-0.983$ & & $0.058-0.485$ & -0.559 to 0.181 \\
DL-R & $0.129(0.019)$ & $0.145(0.019)$ & & $-0.955(0.018)$ \\
& $0.093-0.166$ & $0.108-0.182$ & & -0.974 to 0.929 \\
EL-H & $-0.194(0.018)$ & $-0.213(0.018)$ & $-0.938(0.002)$ & \\
& -0.229 to 0.157 & -0.248 to 0.177 & -0.941 to 0.935 & \\
\hline
\end{tabular}

2015). Although the changes in body shape were smaller than those observed for growth performance, depth to length ratio (DL-R) had a tendency to increase and EL-H to decrease after five generations of selection. The direction of the changes in both traits show an increasing trend in rotund body shape of the fish. This is desired because as the selection program proceeds in the longer term, the fish would become rounder and probably have greater fillet yield. The positive genetic trend in these two measures of body shape are expected as predicted from the estimates of genetic correlations between DL-R (EL$\mathrm{H}$ ) and body weight or daily gain (Table 3 ). Similar genetic correlation estimates have also reported in other fishes by Trọng et al. (2013) and Blonk et al. (2010).

In addition to the estimation of genetic changes in body shape as a result of the selection program for high growth, we estimated heritability and common full-sib effect for these traits. Considerable additive genetic variation was observed for both measures of body shape, indicating that they can be explored by selection. The heritabilities for DL-R and EL-H obtained from our study fall within the range $(0.08-0.23)$ reported in the literature for various body-shape definitions across farmed aquaculture species (Gjerde and Schaeffer 1989; Blonk et al. 2010; Haffray et al. 2013; Trọng et al. 2013; Komen et al. 2014). The present population still show abundant genetic variation for body traits (weight and daily gain), and there is no evidence of reduction in genetic variation as compared with our earlier results estimated in the first two generations (2008-2009) (Santos et al. 2011). The heritabilities estimated for growth traits in our study are comparable

Table 4 Genetic trends and genetic gain estimated for growth and body shape traits

\begin{tabular}{lll}
\hline Traits & Regression equation & Genetic gain $(\%)$ \\
\hline Weight & $\mathrm{Bv}=-53.353+22.4011 \mathrm{~g}$ & 4.08 \\
Daily gain & $\mathrm{Bv}=-0.1857+0.0782 \mathrm{~g}$ & 4.34 \\
DL-R & $\mathrm{Bv}=-0.0003+0.0002 \mathrm{~g}$ & 0.32 \\
EL-H & $\mathrm{Bv}=0.0007-0.0004 \mathrm{~g}$ & -0.09 \\
\hline
\end{tabular}

$\mathrm{g}=$ generation; $\mathrm{Bv}=$ Breeding Value with those reported in the GIFT strain under freshwater pond culture (Nguyen et al. 2010; Ponzoni et al. 2011; Hamzah et al. 2014) or freshwater cages (Trọng et al. 2013) as well as in brackish water systems of moderate salinity (Ninh et al. 2014).

In our present study, the common family effect $\left(c^{2}\right)$ was greater for growth-related traits than for measures of body shape (0.07-0.11 vs 0.03-0.04). Our $\mathrm{c}^{2}$ estimates had similar magnitude to those reported by Nguyen et al. (2010), but were considerably smaller than the $\mathrm{c}^{2}$ values of Nguyen et al. (2007), Ponzoni et al. (2011) and Hamzah et al. (2014). In this study and the one from Nguyen et al. (2010), the fish were harvested at an average body weight of $560 \mathrm{~g}$, whereas it was only 250-300 g in the latter studies (Nguyen et al. 2007; Ponzoni et al. 2011; Hamzah et al. 2014). In agreement with our findings regarding the small common family effect for body shape, other studies in Nile tilapias, using multi-trait models (Yoshida et al. 2013) and random regression analysis (Rutten et al. 2005; Turra et al. 2012; Conti et al. 2014), reported that common family effect is more important in early than later phases of growth.

The weak genetic associations between body shape and growth traits are consistent with the slight changes in these traits as a consequence of selection for high growth (see above Discussion). However, Trọng et al. (2013) found a moderate to high and positive genetic correlations between body measurements (length, height, and thickness) and EL-H $(\mathrm{rg}=0.24-0.62)$. A negative genetic correlation between body height and EL-H were reported in common sole by Blonk et al. (2010). In rainbow trout, Kause et al. (2003) found a strong genetic association between body shape and body mass, and the authors suggested that selection to increase growth rate may promote changes in body shape and that there is a tendency for the fish to become rotund.

As expected in both terrestrial and aquatic animal species, the genetic correlation between body weight and daily gain is high and positive, which are consistent with the positive genetic trend for the former trait (weight) as a result of selection on daily gain. The strong genetic association between the two traits (weight and daily gain) has also been reported in other 
Nile tilapia populations (Kunita et al. 2013; Trọng et al. 2013; Yoshida et al. 2013). For body-shape traits, DL-R was genetically correlated negatively with EL-H, reflecting the fact that only one of the two traits should be used in practical breeding programs.

We derived measures of body shape from morphometric body dimensions. This method is widely applied across farmed aquaculture species, as the information about growth and body traits are often recorded on a routine basis in selective breeding programs. However, improvement of ratio traits is difficult because they are known to lead to statistical hurdles. This is the likely disproportionate nature by which selection pressure is exerted on component traits, hence resulting in unpredictable changes in either the numerator or denominator (Gunsett 1984). Objective measurements using image technologies would provide information to calculate body shape of the animals (Adams et al. 2013). In addition to new developments of computer software packages used to analyse animal images, devised methods of computations/calculations to predict fillet yield and its association with body shape will enable simultaneous improvement in both production and appearance of Nile tilapia.

\section{Conclusion}

Realized correlated response in fish body shape was estimated for the first time in Nile tilapia, indicating that selection for high daily gain had a tendency to produce rotund fish, albeit at a very slow rate. These positive changes resulted from the favorable genetic correlations of growth traits (body weight and daily gain) with depth ratio or with the ellipticity midsagittal plane.

Acknowledgments This study was funded by Conselho Nacional de Desenvolvimento Científico e Tecnológico, Brazil (CNPq) and Ministério de Pesca e Aquicultura, Brazil (MPA). Peixegen Research Group provided the data set. We extend our thanks to the University of the Sunshine Coast for hosting the sabbatical of Prof Carlos Antonio Lopes de Oliveira from January to August 2015, and to Professors Wayne Knibb and Richard Burns who helped to review language of the draft manuscript.

\section{References}

Adams DC, Rohlf FJ, Slice DE (2013) A field comes of age: geometric morphometrics in the 21st century. Hystrix Ital J Mammal 24:7-14

Blonk R, Komen J, Tenghe A, Kamstra A, Van Arendonk J (2010) Heritability of shape in common sole, Solea solea, estimated from image analysis data. Aquaculture 307:6-11

Colihueque N, Araneda C (2014) Appearance traits in fish farming: progress from classical genetics to genomics, providing insight into current and potential genetic improvement. Front Genet 5:251
Conti ACM et al (2014) Genetic parameters for weight gain and body measurements for Nile tilapias by random regression modeling. Semina Ciênc Agrár 35:2843-2858

Costa $C$ et al (2010) Genetic and environmental influences on shape variation in the European sea bass (Dicentrarchus labrax). Biol J Linn Soc 101:427-436

Cowles MK, Carlin BP (1996) Markov chain Monte Carlo convergence diagnostics: a comparative review. J Am Stat Assoc 91:883-904

Gjerde B, Schaeffer L (1989) Body traits in rainbow trout: II. Estimates of heritabilities and of phenotypic and genetic correlations. Aquaculture 80:25-44

Gunsett F (1984) Linear index selection to improve traits defined as ratios. J Anim Sci 59:1185-1193

Haffray P et al (2013) Genetic parameters of in-vivo prediction of carcass, head and fillet yields by internal ultrasound and 2D external imagery in large rainbow trout (Oncorhynchus mykiss). Aquaculture 410: 236-244

Hamzah A et al (2014) Tropical agricultural science. J Trop Agric Sci 37: 411-429

Kause A, Ritola O, Paananen T, Eskelinen U, Mäntysaari E (2003) Big and beautiful? Quantitative genetic parameters for appearance of large rainbow trout. J Fish Biol 62:610-622

Komen H, Blonk R, Sae-Lim P (2014) Genetic analysis of shape in trout, using image analysis. In: Proceedings, 10th World Congress of Genetics Applied to Livestock Production

Kunita N et al (2013) Avaliação genética de características morfométricas em tilápias do Nilo cultivadas. Archivos de Zootecnia 62: $555-566$

Nguyen NH (2015) Genetic improvement for important farmed aquaculture species with a reference to carp, tilapia and prawns in Asia: achievements, lessons and challenges. Fish and Fisheries [In press]

Nguyen NH, Khaw HL, Ponzoni RW, Hamzah A, Kamaruzzaman N (2007) Can sexual dimorphism and body shape be altered in Nile tilapia (Oreochromis niloticus) by genetic means? Aquaculture 272: S38-S46

Nguyen NH et al (2010) Correlated response in fillet weight and yield to selection for increased harvest weight in genetically improved farmed tilapia (GIFT strain), Oreochromis niloticus. Aquaculture 305:1-5

Ninh NH, Thoa NP, Knibb W, Nguyen NH (2014) Selection for enhanced growth performance of Nile tilapia (Oreochromis niloticus) in brackish water (15-20ppt) in Vietnam. Aquaculture 428:1-6

Ponzoni RW et al (2011) Genetic improvement of Nile tilapia (Oreochromis niloticus) with special reference to the work conducted by the WorldFish Center with the GIFT strain. Rev Aquac 3:27-41

Rutten MJ, Komen H, Bovenhuis H (2005) Longitudinal genetic analysis of Nile tilapia (Oreochromis niloticus L.) body weight using a random regression model. Aquaculture 246:101-113

Santos AI et al (2011) Bayesian genetic parameters for body weight and survival of Nile tilapia farmed in Brazil. Pesq Agrop Brasileira 46: $33-43$

SAS Institute (2007) Statistical Analysis System (SAS) SAS Institute, Cary NC, USA

Sorensen D, Gianola D (2002) Likelihood, Bayesian, and MCMC methods in quantitative genetics. Springer, Dordrecht

Trọng TQ, Mulder HA, van Arendonk JA, Komen H (2013) Heritability and genotype by environment interaction estimates for harvest weight, growth rate, and shape of Nile tilapia (Oreochromis niloticus) grown in river cage and VAC in Vietnam. Aquaculture 384:119-127

Turra EM et al (2012) Estimation of genetic parameters for body weights of Nile tilapia Oreochromis niloticus using random regression models. Aquaculture 354:31-37 
Van Tassell C, Van Vleck L (1995) A manual for use of MTGSAM. A set of Fortran programs to apply Gibbs sampling to animal models for variance component estimation. US Department of Agriculture, Agricultural Research Service

Weatherley AH, Gill H, Casselman JM (1987) The biology of fish growth. Academic Press, London
Whatmore P et al (2013) Genetic parameters for economically important traits in yellowtail kingfish Seriola lalandi. Aquaculture 400:77-84

Yoshida GM et al (2013) Associação entre características de desempenho de tilápia-do-nilo ao longo do período de cultivo. Pesq Agrop Brasileira 48:816-824 